\title{
Europas Bevölkerung verlangt Selbstbestimmung am Lebensende
}

\author{
Die Vereinigung Schweizer Medizinalrechtsanwälte (SMLA) hat den Eindruck, die in \\ Europa geltenden Rechtsordnungen zur selbstbestimmten Beendigung des Lebens \\ entsprächen nicht mehr dem Willen grosser Teile der Bevölkerung. Zur Überprüfung \\ hat sie eine Umfrage in mehreren europäischen Ländern in Auftrag gegeben.
}

\section{Frank Th. Petermann}

Rechtsanwalt, Präsident der Vereinigung Schweizer Medizinalrechtsanwälte in Zürich

Es bestehen keine Interessenbindungen.

Korrespondenz:

Dr. iur. Frank Th. Petermann c/o Vereinigung der Schweizer Medizinalrechtsanwälte Seebahnstrasse 85 CH-8003 Zürich Tel. 0445860950 Fax 0445860955

info[at]medizinalrecht.org www.medizinalrecht.org
Im Herbst 2012 führte die Vereinigung der Schweizer Medizinalrechtsanwälte (SMLA) über das zur Gallup-Gruppe gehörende Meinungsforschungsinstitut ISOPUBLIC eine Online-Umfrage in zwölf europäischen Ländern zum Thema der selbstbestimmten Beendigung des Lebens durch. Die Rücklaufquote (Bruttoausschöpfung) betrug 37 Prozent.

\section{Hintergrund der Studie}

Hintergrund dieser Studie war zunächst einmal, dass die SMLA in diesem Bereich bereits seit längerer Zeit Diskrepanzen zwischen Recht, Politik und Gesellschaft beobachten konnte. Die fünf wichtigsten dieser Diskrepanzen sind:

- die Aussage im Urteil «Haas gegen die Schweiz» vom 20. Januar 2011 des Europäischen Gerichtshofes für Menschenrechte (EGMR), wonach jeder Mensch über die Frage, wann und wie er sterben möchte, selber entscheiden darf (anerkannt als Bestandteil des Selbstbestimmungsrechts gemäss Art. 8 der Europäischen Menschenrechtskonvention);

- der vom selben Gerichtshof erfolgte Hinweis, dass in 42 untersuchten Staaten des Europarates nur vier - die Benelux-Staaten und die Schweiz eine Möglichkeit kennen, Sterbehilfe durch Beihilfe zum Suizid zu leisten (Urteil «Koch gegen Deutschland» vom 19. Juli 2012);

- der Umstand, dass in den meisten europäischen Staaten die Beihilfe zum Suizid seit etwa dem 19. Jahrhundert unter Strafe steht;

- die Beobachtung, dass in praktisch allen europäischen Ländern viele Anzeichen dafür sprechen, dass die geltende Rechtsordnung in dieser Hinsicht nicht mehr dem Willen grosser Teile der Bevölkerung entspricht;

- dass vor Gerichten verschiedener europäischer (und anderer!) Staaten Verfahren von Bürgern anhängig gemacht wurden, die darauf abzielen, vom Staat errichtete rechtliche Barrieren auszuräumen, die ihnen den Weg zu einer selbstbestimmten Beendigung des eigenen Lebens versperren.

\section{La population européenne demande le droit à l'auto- détermination en fin de vie}

A l'automne 2012, la Fédération des avocats suisses du droit médical (SMLA) a mandaté I'Institut de sondage ISOPUBLIC, rattaché au groupe GALLUP, pour réaliser une enquête en ligne sur le thème de l'autodétermination en fin de vie dans 12 pays d'Europe. Les importants clivages existant dans ce domaine depuis de nombreuses années entre l'appréciation juridique, le monde politique et la société, et ce tant en Suisse qu'à l'étranger, ont donné lieu au lancement de cette étude. Comme la Convention européenne des droits de l'Homme (CEDH) intervient également dans le volet juridique de cette question, il a été relativement vite décidé d'étendre l'enquête à d'autres pays européens. En effet, les conclusions recueillies pourraient également servir lors de l'interprétation de questions supplémentaires relevant du droit médical (par ex. dans le domaine du human enhancement, de la médecine du sport, etc.). L'enquête a confirmé qu'une nette majorité de la population européenne souhaitait avoir accès à l'assistance au suicide. De plus, les résultats obtenus apportent une aide importante pour l'interprétation de l'art. 8, al. 2 de la CEDH, et notamment pour comprendre la notion de «société démocratique» au sens de ce même article. Par ailleurs, le présent article aborde brièvement de nombreuses questions soulevées par l'enquête sur le rôle du corps médical dans ce domaine. 


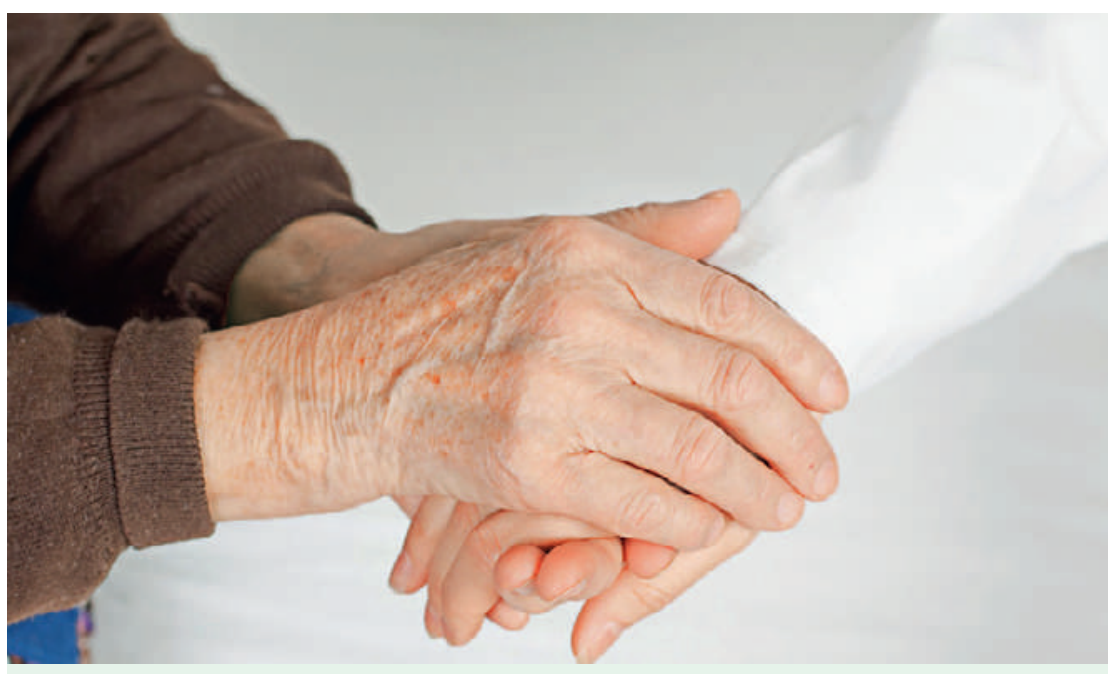

Gefragt wurde auch, ob es Ärzten verboten sein soll, im Zusammenhang mit Sterbehilfe tätig zu sein.
* Es wurde mit einem Signifikanzniveau von $95,5 \%$ gerechnet. Die ausgewiesene Signifikanz bedeutet, dass ein wissenschaftlich nachweisbarer Unterschied zwischen den untersuchten Gruppen besteht.
Angesichts dieser z.T. erheblichen Diskrepanzen entschloss sich die SMLA, bei einem renommierten Forschungsinstitut eine Arbeit in Auftrag zu geben, welche die Haltung der Bevölkerung zu diesen Fragen untersucht. Da die betroffene Materie nicht nur durch schweizerisches Verfassungsrecht, sondern auch durch Völkerrecht (die Europäische Menschenrechtskonvention von 1950, EMRK) geregelt ist, erschien es von Anfang an sinnvoll, die Umfrage nicht in der Schweiz oder den anderen europäischen Ländern, die liberale Regelungen kennen, sondern in anderen europäischen Ländern durchzuführen. Die Umfrage wurde sodann in zwölf europäischen Ländern durchgeführt, in denen liberale Regelungen bislang fehlen. Diese verhältnismässig umfangreiche Befragung rechtfertigte sich u.a. auch deswegen, weil die daraus gewonnenen Erkenntnisse mutatis mutandis für andere medizinalrechtliche Fragestellungen (so etwa im Bereich Enhancementmedizin, Sportmedizin usw.) wichtige Auslegungshilfen bieten können.

\section{Ergebnisse der Studie}

Befragt wurden vom 24. September bis zum 9. Oktober 2012 mindestens je 1000 Personen in Dänemark, Deutschland, Finnland, Frankreich, Griechenland, Grossbritannien, Irland, Italien, Österreich, Portugal, Schweden und Spanien. Damit sind nach den Regeln der Meinungsforschung die Ergebnisse repräsentativ. Die Teilnehmer wurden mittels telefonischer und persönlicher Interviews vorrekrutiert. Die Stichprobenstruktur der Studie entspricht der Bevölkerungsstruktur. Sie kann auf der Webseite der Vereinigung der Schweizer Medizinalrechtsanwälte unter der Rubrik Publikationen/Stellungnahmen heruntergeladen werden (www.medizinalrecht.org/ index.php?id=16). Folgende Bereiche sollen hier kurz dargelegt werden:

\section{Deutliche Bejahung der Selbstbestimmung}

Zuerst wurde danach gefragt, ob es richtig sei, dass jeder Mensch selber darüber bestimmen darf, wann und wie er sterben will, oder ob dies nicht dem Einzelnen überlassen bleiben solle. In allen Ländern stimmte eine Mehrheit der Selbstbestimmung zu; in Griechenland mit 52 Prozent, in den restlichen Ländern mit Mehrheiten von 71 Prozent (Dänemark) bis 87 Prozent (Deutschland). Interessanterweise differieren die Antworten bezüglich der Altersgruppen kaum.

Die Auffassung dieser Mehrheiten entspricht einerseits der Auffassung des Europäischen Gerichtshofes für Menschenrechte in Strassburg. Dieser hat am 20. Januar 2011 im Fall «Haas gegen die Schweiz» geurteilt, das Recht, selber zu entscheiden, wann und wie man sterben wolle, sei Bestandteil des Selbstbestimmungsrechts und damit menschenrechtlich geschützt. Allerdings entspricht das Strafrecht in den meisten europäischen Staaten dem nicht, stellt es doch meist Beihilfe zum Suizid unter Strafe.

\section{Selber Sterbehilfe beanspruchen?}

Die zweite Frage erkundigte sich danach, ob jemand sich vorstellen könne, selbst Sterbehilfe in Anspruch zu nehmen, falls er oder sie an einer unheilbaren, schweren Krankheit, an schwerer Invalidität oder nicht beherrschbaren Schmerzen leide, oder ob dies für die befragte Person nicht in Frage komme.

Auch in dieser Frage finden sich in sämtlichen Ländern Mehrheiten: 56 Prozent der Griechen, 68 Prozent der Iren, bis zu 77 Prozent der Deutschen und gar 78 Prozent der Spanier können sich vorstellen, selbst einmal bei entsprechend schlechter Gesundheit Sterbehilfe in Anspruch zu nehmen. Dabei zeigt sich nur in Frankreich (mit 75 Prozent) ein signifikanter* Unterschied zwischen den Altersgruppen. Unter 34 und über 55 Jahre alte Befragte stimmten mit 71 Prozent zu, 35 bis 54 Jahre alte Personen jedoch mit 82 Prozent.

\section{Verlangt wird professionelle Sterbehilfe}

Mit dieser Frage sollte geklärt werden, ob Sterbehilfe ausschliesslich mit «professioneller Hilfe - d.h. mit einem Arzt oder einem ausgebildeten Sterbehelfer»möglich sein sollte, oder ob Sterbehilfe auch ohne professionelle Begleitung durchgeführt werden solle. Hier variieren die Mehrheiten zwischen 76 Prozent (Griechenland) und 89 Prozent (Schweden) für Professionalität. In den beiden Ländern mit der grössten Zustimmung (Spanien und Deutschland, Letzteres mit 80 Prozent) ergaben sich dabei die grössten Unterschiede zwischen den Altersgruppen. Je jünger die Befragten, desto höher die Forderung nach professioneller Sterbehilfe: unter 34-Jährige 86 bzw. 88 Prozent, 35- bis 54-Jährige 74-78 Prozent, über 54-Jährige 78 und 76 Prozent.

\section{Strafandrohungen für professionelle Sterbehilfe massiv abgelehnt}

In allen befragten Ländern - ausgenommen in Deutschland - gibt es Gesetze, die Beihilfe zum Freitod mit Strafe bedrohen. Vor dem deutschen Bun- 
destag liegt ein Antrag der deutschen Regierung, «gewerbsmässige Vermittlung von Gelegenheiten zur Selbsttötung» für strafbar zu erklären. Es wurde nach der Meinung zu solchen Strafandrohungen gefragt. Auch hier ergaben sich klare Mehrheiten gegen derartige Staatseingriffe; sie reichen von 58 Prozent (Schweden) bis 82 Prozent (Spanien sowie Portugal). Die Deutschen lehnen das geplante Gesetz, welches eine solche Strafbarkeit einführen will, gar mit 76 Prozent $a b$.

\section{Für die Freiheit der Ärzte, helfen zu dürfen}

An fünfter Stelle wollte die Umfrage klären, ob es Ärzten verboten sein soll, im Zusammenhang mit Sterbehilfe tätig zu sein. Ein derartiger Eingriff in die Entscheidungsfreiheit der Ärzte wurde in allen Ländern mit deutlicher bis grosser Mehrheit abgelehnt. Die Befragten in Griechenland setzten sich mit 58 Prozent für die Freiheit der Ärzte ein, jene in Grossbritannien mit 84 Prozent. In Deutschland, wo die Bundesärztekammer für das ärztliche Berufsrecht eine Verbotsbestimmung fordert, wird dies von 79 Prozent der Befragten abgelehnt. gleichzeitig in mehreren Staaten Europas stattfand; so konnte das erste Mal länderübergreifend verglichen werden. Die repräsentative Umfrage hat bestätigt, was das Abstimmungsresultat der beiden EDUEVP-Volksinitiativen aus Zürich am 15. Mai 2011 bereits stark vermuten liess: Die grosse Mehrheit der Bevölkerung in Europa will selbst über Art und Zeitpunkt der Beendigung des eigenen Lebens entscheiden können (oder dürfen).

\section{Auslegungshilfe für Art. 8 EMRK \\ (demokratische Gesellschaft)}

Dieses Ergebnis stellt sodann eine wichtige Auslegungshilfe für Art. 8 Abs. 2 EMRK dar. Das Recht, selbst über Art und Zeitpunkt des eigenen Todes zu entscheiden, ist Ausfluss des in Art. 8 Abs. 1 EMRK statuierten Rechts auf Achtung des Privat- und Familienlebens. Absatz 2 von Art. 8 EMRK erlaubt den Vertragsstaaten unter ganz bestimmten Umständen, diese Rechte $\mathrm{zu}$ beschneiden. Allerdings nur unter der Grundvoraussetzung, dass solche Eingriffe «in einer demokratischen Gesellschaft» für den Schutz anderer Polizeigüter notwendig sind. Die Formulie-

\section{«Die grosse Mehrheit der Bevölkerung in Europa will selbst über Art und Zeitpunkt der Beendigung des eigenen Lebens entscheiden können.»}

\section{Befürchtung, es könnte Druck auf Alte und Kranke geben?}

Die sechste Frage wandte sich dem Argument zu, das häufig insbesondere von Gegnern der Sterbehilfe aus kirchlichen Kreisen angeführt wird, nämlich: Wenn Sterbehilfe erlaubt werde, könnten sich Alte und Kranke unter Druck gesetzt fühlen, die Sterbehilfe in Anspruch zu nehmen, um dem System und ihren Angehörigen nicht zur Last zu fallen. Gefragt wurde danach, «ob jemand wie Sie unter Druck gesetzt werden könnte, um möglichst schnell den Freitod zu wählen». Dabei konnten die Befragten vier Stufen wählen, nämlich es könnte «häufig», «ab und zu», «selten» oder «praktisch nie» zu solchen Druckversuchen kommen. Mit Ausnahme von Griechenland und Spanien haben die Befragten in allen Ländern die Wahrscheinlichkeit eines solchen Drucks mehrheitlich als wenig wahrscheinlich eingestuft.

\section{Schlussfolgerungen aus der Studie}

\section{Bestätigung, dass die Bevölkerung} «Sterbehilfe will»

Meinungsumfragen in diesem Bereich wurden bereits seit Jahren in einzelnen Ländern durchgeführt. Ihre Ergebnisse waren mit denen der jetzt durchgeführten Studie im Wesentlichen kongruent. Die Studie der SMLA ist insofern ein Novum, als dass sie rung «in einer demokratischen Gesellschaft» legt nahe, dass eine Einschränkung nicht nur legal im eigentlichen Sinn des Wortes zu sein hat, sondern auch einer demokratischen Legitimierung bedarf. Es ist also nicht nur zu prüfen, ob sich entsprechende Verbote auf im formellen Sinn im richtigen Verfahren erlassene Gesetze stützen, sondern auch, ob eine Mehrheit (der Gesellschaft) ein vor Zeiten erlassenes, damals demokratisch zustande gekommenes Gesetz in ihrer Mehrheit überhaupt noch billigt. Mit anderen Worten, die Einschränkung muss auch dem Willen der Mehrheit der aktuell lebenden Gesellschaft entsprechen, denn das ist es, was man im deutschen Sprachgebrauch unter dem Begriff «Demokratie» versteht.

\section{Rolle der Ärzteschaft in diesem Bereich}

Im Bereich der selbstbestimmten Beendigung des eigenen Lebens spielen Ärzte und Ärzteschaft (gemeint sind damit insbesondere Standesorganisationen) eine wichtige Rolle. Diese Rolle ist jedoch bereits in einem Umbruch begriffen. Es seien an dieser Stelle nur drei nach Meinung des Autors besonders wichtige Punkte genannt:

\section{Vertrauensverhältnis zum Patienten}

Mehr als jedes andere Auftragsverhältnis beruht die Arzt-Patienten-Beziehung auf einem Vertrauensver- 
hältnis. Theoretisch besteht das Vertrauensverhältnis in beide Richtungen, faktisch natürlich in erster Linie vom Patienten zum Arzt. Es stellt sich die Frage, ob dieses Vertrauensverhältnis nicht darunter leidet, wenn zwischen Patient und Arzt stark unterschiedliche Vorstellungen über existentielle Fragen von Leben und Sterben bestehen, selbst wenn der Arzt ohne innere Überzeugung nur das nach Aussen vertritt, was die Ärzteschaft von ihm verlangt. Das von der Ärzteschaft häufig ins Feld geführte Argument, das Vertrauensverhältnis zwischen Arzt und Patient werde belastet, wenn der Patient weiss, dass der Arzt auch bei einer selbstbestimmten Beendigung des Lebens behilflich wäre, kann gerade mit Wissen um diese Umfrageergebnisse nur noch schwer nachvollzogen werden. Im Ausland wurde diese Thematik längst wissenschaftlich untersucht [1] und das Ergebnis der Studie lässt sich dahingehend zusammenfassen, dass die Arzt-PatientenBeziehung von einer offenen Diskussion über die selbstbestimmte Beendigung des eigenen Lebens profitiert. gesellschaften und kantonale Ärztegesellschaften stützen sich auf die Richtlinien der SAMW, die jedoch nur für jene seltenen Fälle erlassen worden sind, in denen Patienten von Ärzten diese kurz vor dem natürlichen Ableben bitten, ihre restliche Leidenszeit verkürzen zu helfen. Insoweit diese Richtlinien darüber hinaus Ärzten etwas verbieten wollen, was gegenüber anderen Bürgern nicht (und vor Gericht wohl auch Ärzten gegenüber nicht) durchsetzbar ist, sind sie aber völkerrechts- und verfassungswidrig. Deshalb wird die SAMW nicht umhinkommen, ihre Haltung in diesem Bereich zu überdenken und allenfalls $\mathrm{zu}$ revidieren. Sie hat verschiedene Möglichkeiten, dies zu tun. Sie kann dies verhältnismässig rasch und unter Ausnützung eines jetzt noch vorhandenen Bewegungsspielraums tun, oder aber noch einige Jahre warten - und dann den Gerichten die Ausgestaltung ihrer Rolle überlassen. Ob Letzteres die bessere Lösung ist, auch bezüglich der Rolle, die sie als wichtiger Faktor im Gesundheitswesen diesen gegenüber hat, muss sie letztendlich selbst beantworten.

«Deshalb wird die SAMW nicht umhinkommen, ihre Haltung in diesem Bereich zu überdenken.»

\section{Auch Ärzte sind Bürger - auch sie haben ein Recht auf Achtung ihres Privat- und Familienlebens}

Wenn eine repräsentative Umfrage ergibt, dass eine grosse Mehrheit der Bürger selbst über Art und Zeitpunkt des eigenen Lebens entscheiden will, so liegt die Vermutung nahe, dass auch Ärzte dies wünschen. Wenn sie sich dies für sich selbst wünschen, so liegt auch der Schluss nahe, dass sie wünschen, ihren Patienten in diesen Momenten beistehen zu können. Hier kommt Art. 8 Abs. 1 EMRK (Recht auf Achtung des Privat- und Familienlebens) erneut zum Tragen, allerdings in einer anderen Funktion. Denn zur Achtung des Privat- und Familienlebens gehört (neben dem Recht, selbst über Art und Zeitpunkt des eigenen Todes zu bestimmen) auch das Recht, bei der Ausübung seines Berufs nicht daran gehindert zu werden, diesen innerhalb der rechtlichen Leitplanken des Rechtsstaats auszuüben. Das heisst nichts anderes, als dass nicht nur Patienten auf die Beseitigung von grundrechtswidrigen rechtlichen Barrieren klagen können, sondern selbstverständlich auch Ärzte.

\section{Künftige Rolle der Ärzteschaft}

Die Organisationen der Ärzteschaft als Vertreter der Ärzte haben auch in der Schweiz eine eher restriktive Haltung eingenommen. Die FMH, viele Fachärzte-
Die Vereinigung der Schweizer Medizinalrechtsanwälte hat mit dieser Studie einige der sie in diesem Bereich interessierenden Fragestellungen beantworten können. Die Ergebnisse der Studie sind international einem breiten Kreis von Wissenschaft, Politik und Medien bekanntgemacht worden. Auf diese Weise soll vor allem auch den politisch Verantwortlichen ermöglicht werden, bei allfälligen gesetzgeberischen Überlegungen $\mathrm{zu}$ diesen Themen dem demokratischen Prinzip Rechnung zu tragen.

\section{Literatur}

1 Anthony L. Back AL, Starks H, Hsu C, Gordon JR, Bharucha A, Pearlman RA. Clinician-Patient Interactions About Requests for Physician-Assisted Suicide. A Patient and Family View. In: Arch Intern Med. 2002;162:1257-65)

\section{Interaktiver Artike}

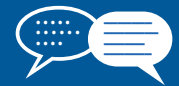

Wollen Sie diesen Artikel kommentieren? Nutzen Sie dafür die Kommentarfunktion in der OnlineVersion oder sehen Sie nach, was Ihre Kolleginnen und Kollegen bereits geschrieben haben: www.saez.ch/aktuelle-ausgabe/interaktive-beitraege/ 